\section{Origin of Cosmic Ray Stars}

As yet, the absorption of the star-producing radiation has only been measured in air. Nevertheless, the variation of the intensity of single tracks recorded in photographic plates under various lead absorbers has been measured at high altitude and at sea-level ${ }^{\mathbf{1 , 2}}$. The decrease of single tracks with a given lead absorber is found to be much less than the decrease of stars in an equal weight of the atmosphere. Perkins ${ }^{2}$ has shown that the number of single tracks is proportional to the number of stars, and therefore it appears established that the range of the star-producing component of cosmic radiation expressed in $\mathrm{gm} . / \mathrm{cm} .{ }^{2}$ is larger by a factor of approximately 3 in lead than it is in air.

This difference in range could be caused possibly by the difference in $(a)$ density, or $(b)$ atomic weight, $A$, of the two materials. Perkins has assumed the former and has concluded that the stars are produced by an unstable particle. It seems worth while to explore the latter possibility, as this leads to a dependence of the range of the primaries on $A$, not very dissimilar from that found experimentally.

Thus, it is well known that the nuclear radius is approximately proportional to $A^{1 / 3}$ :

$$
R=r_{0} A^{1 / 3} \text {, with } r_{0} \sim 10^{-13} \mathrm{~cm} .
$$

The cross-section, $\sigma$, will be $\pi R^{2}$, and the number of nuclei per gm./cm. ${ }^{2}$, $n$, will be $N / A$, where $N$ is Avogadro's number. Hence the mean free path for a radiation interacting strongly with the nuclei will be given by:

$l=1 / n \pi R^{2} \mathrm{gm} . / \mathrm{cm} .{ }^{2}$, or $l=A^{1 / 3} / N \pi r_{0}{ }^{2} \mathrm{gm} . / \mathrm{cm} .{ }^{2}$.

Substituting numerical values, one obtains:

$$
l_{\text {air }}=130 \mathrm{gm} . / \mathrm{cm}^{2}, l_{\text {lead }}=311 \mathrm{gm} . / \mathrm{cm}^{2},
$$

which do not differ greatly from the observed values.

Thus, as an alternative to the assumption of unstable primaries, one may equally well assume the primaries to be stable particles having a cross-section for star production given by the so-called geometric cross-section, and hence a range given by (2).

From the known constitution of the Ilford nuclear emulsion, one obtains for the range of the primaries in the emulsion on this basis :

$$
l_{E}=200 \mathrm{gm} . / \mathrm{cm}^{2}=50 \mathrm{~cm} .,
$$

since $P E=4$. Let $S$ be the number of primaries crossing $1 \mathrm{~cm} .^{2}$ per day, and $I$ the number of stars formed per c.c. per day. Then approximately :

$$
S / l_{E}=I \text {. }
$$

Now, at Jungfraujoch, $I \sim 10$ stars/c.c./day, and hence :

$$
S \sim 500 / \mathrm{cm}^{2} / \mathrm{day}
$$

or, in other words, about 12 per cent of the total corpuscular radiation at this altitude.

This is in rough agreement with Schopper's estimate $^{3}$ of the intensity of fast neutrons, based on meas urements of the number of recoil protons recorded in plates covered with paraffin. Thus, the assumption that the majority of cosmic ray stars are caused by fast neutrons is not in violent disagreement with the existing data.

Physics Department, Birkbeck College,

$$
\text { E. P. George }
$$

London, E.C.4. May 19.

1 Heitler, Powell and Heitler, Nature, 146, 65 (1940).

? Perkins, Nature, 160, 707 (1947).

' Schopper, Phys. Z., 40, 22 (1939).

\section{Random Association of Points on a Lattice}

A LATTICE is defined to be a rectangular array of points each of which may be any one of $k$ colours. In a previous communication ${ }^{1}$, I have given the first and the second moments for the probability distribution of the total number of joins between points of different colours, when the probability that any point is of colour $r$ is $p_{r}\left(\sum_{1}^{k} p_{r}=1\right)$ and is independent of the colour of all the other points. A join was there defined as a line between two adjacent points parallel to the axes of the lattice. The present note gives the first and the second moments for the distribution of (1) the number of joins between points of the same colour, and (2) the total number of joins between points of different colours for two- and three-dimensional lattices when all possible joins between adjacent points are included. The first distribution corresponds to Todd's distribution of 'doublets'2.

(1) Joins between points of the same colour, say, rth colour.

(a) Two-dimensional lattice: $m \times n$ points :

$$
\begin{aligned}
\mu_{1}{ }^{\prime}= & (4 b-3 a+2) p_{r^{2}}, \\
\mu_{3}= & (4 b-3 a+2) p_{r^{2}} \\
& +8(7 b-9 a+11) p_{r}{ }^{2} \\
& -15(4 b-5 a+6) p_{r}
\end{aligned}
$$

(b) Three-dimensional lattice : $l \times m \times n$ points :

$$
\begin{aligned}
\mu_{1}^{\prime}= & (13 e-9 d+6 c-4) p_{r^{2}}, \\
\mu_{2}= & (13 e-9 d+6 c-4) p_{r^{2}} \\
& +2(325 e-378 d+432 c-488) p_{r^{3}} \\
& -(663 e-765 d+870 c-980) p_{r^{4}} .
\end{aligned}
$$

(2) Total number of joins between points of different colours.

(a) Two-dimensional lattice: $m \times n$ points :

$$
\begin{aligned}
\mu_{1}^{\prime}= & 2(4 b-3 a+2) \Sigma p_{r} p_{s}, \\
\mu_{2}= & 2(32 b-39 a+46) \Sigma p_{r} p_{s} \\
& =8(39 b-48 a+57) \Sigma p_{r} p_{s} p_{t} \\
& =60(4 b-5 a+6) \Sigma p_{r}^{2} p_{s}^{2} \\
& +120(4 b-5 a+6) \Sigma p_{r} p_{s} p_{t} p_{u} .
\end{aligned}
$$

(b) Three-dimensional lattice : $l \times m \times n$ points :

$$
\begin{aligned}
\mu_{1}^{\prime}= & 2(13 e-9 d+6 c-4) \Sigma p_{r} p_{s}, \\
\mu_{2}= & 2(338 e-387 d+438 c-492) \Sigma p_{r} p_{s} \\
& -2(1677 e-1926 d+2184 c-2456) \Sigma p_{r} p_{s} p_{t} \\
& -4(663 e-765 d+870 c-980) \Sigma p_{r} p_{s}{ }^{2}
\end{aligned}
$$$$
+8(663 e-765 d+870 c-980) \Sigma p_{r} p_{s} p_{t} p_{u} \text {. }
$$
In all these expressions, $a=m+n, b=m n, c=l+m+n, d=$
$l m+m n+n l$, and $e=l m n$. The summations are taken for all values of $r, s, t$ and $u$ such that $1 \leqslant r<8<t<u \leqslant k$.

All the distributions tend to the normal form when $l, m$ and $n$ tend to infinity.

The corresponding results when the lattices consist of a fixed number of points of the various colours, say $n_{1}, n_{2}, \ldots n_{k}$, have also been worked out. These results, being long and complicated, will be given in another communication to be published shortly.

Department of the Design and

$$
\text { P. V. Krishna IYer }
$$

Analysis of Scientific Experiment, Oxford. April 9.

${ }^{1}$ Krishna Iyer, P. V., Nature, 160, 714 (1947).

${ }^{2}$ Todd, H., J. Roy. Stat. Soc. Suppl., 7, 78 (1940). 